\title{
artículos
}

\section{I progetti della conoscenza e del restauro per la conservazione e la valorizzazione delle architetture e degli oggetti d'arte}

\author{
Antonio Pugliano \\ Università "Roma Tre"
}

RESUMEN

El presente artículo versa sobre los orígenes culturales y los antecedentes históricos que constituyen la base de la metodología proyectual de la restauración, enfocada a la valorización del patrimonio. Dicha metodología comporta una redefinición del actual concepto de restauración, acorde con las nuevas exigencias que plantea el uso y disfrute de los bienes culturales. El método restaurador al cual nos referimos y que exponemos en este trabajo a través de algunos ejemplos reales, debe resultar útil para la valorización de la arquitectura $y$ de las obras de arte, las cuales deben ser conservadas correctamente en la plenitud de sus valores y significados.

PALABRAS CLAVE: restauración/ conservación/valorización.

The projects of the knowledge and the restoration for the conservation and valuation of architectures and art objects

ABSTRACT

This memory concerns the cultural origins and historical conditions on which the planning methodology for the "restoration aimed at valorization" are based on. That methodology involves the reworking of the restoration model answering to the new requests coming from the use of cultural heritage. Some real examples are addressed to demonstrate the reference method aimed at usefully and properly "restoring for valorizing" architecture and art objects to be stored in the fullness of their values and meanings.

KEY WORDS: restoration/ conservation/valorization.

Premessa.

II restauro è una disciplina scientifica e tecnica che, sempre più, si applica tanto alla mitigazione dei rischi di degrado e danneggiamento cui sono esposte le opere del passato quanto alla risoluzione dei problemi tecnici legati al mantenimento delle qualità materiali ed espressive dei manufatti, ai fini della loro più efficiente fruizione. Questa condizione di esercizio è resa necessaria da un fenomeno pernicioso non sufficientemente considerato nonostante la sua diffusione e pericolosità: la perdita di significato linguistico, di senso, di emblematicità, indotta nelle opere del passato da restauri o manutenzioni inappropriati. Tale fenomeno è molto esteso allo stato attuale poiché la disciplina del restauro, negli esiti, è divenuta espressione del rapporto che

* PUGLIANO, Antonio: "I progetti della conoscenza e del restauro per la conservazione e la valorizzazione delle architetture e degli oggetti d'arte", en Boletín de Arte ${ }^{\circ}$ 32-33, Departamento de Historia del Arte, Universidad de Málaga, 2011-2012, págs. 567-589. Fecha de recepción: Septiembre 2011. 
gli architetti contemporanei, piuttosto che le comunità sociali, tendono a stabilire con il passato, attraverso i loro interventi progettuali applicati ai contesti architettonici e urbani preesistenti. Si tratta di un rapporto complesso che ospita le diverse tendenze della cultura architettonica in un intervallo che il comune sentire, semplicisticamente, comprende tra due limiti opposti: la volontà di porsi in continuità con le opere del passato o, viceversa, l'intenzione di rivendicare l'autonomia, assumendo un atteggiamento d'indifferenza nei riguardi dei caratteri della preesistenza. Non c'è chi non veda quanto sia necessaria una visione diversa, attenta a porre di nuovo, al centro dell'operatività dell'architetto, lo studio approfondito e critico delle opere per ottenere la massima comprensione della loro consistenza storica, linguistica e materiale da parte della comunità sociale. Nella prospettiva citata il progetto di restauro non può che coincidere con il progetto della conoscenza dalla quale si originano, motivatamente, le scelte tecniche. La presente memoria tende a delineare, attraverso una casistica di esempi reali, le linee generali del metodo cui è utile riferirsi, nella condizione attuale, per il restauro di architetture e oggetti d'arte da conservare appropriatamente nella pienezza dei loro valori e significati.

\section{LA FORMAZIONE DEL CONCETTO DI RESTAURO.}

II restauro delle architetture e degli oggetti d'arte ha espresso i termini del rapporto che le comunità sociali hanno ritenuto di dover avere con il proprio passato: conservare, valorizzando le tracce materiali della propria storia,è servito a selezionare le espressioni di cultura che, di volta in volta nei diversi periodi storici, potevano svolgere un ruolo rappresentativo e identitario.Questa chiave di lettura è utile a comprendere le motivazioni dei diversi approcci che si sono succeduti nella storia della conservazione delle tracce del passato.

\section{MODI STORICI DELL'ATTUALIZZAZIONE.}

La maturazione del concetto di valore storico delle opere del passato ha determinato la formazione di un'idea di restauro rigoroso e consapevole; il processo si è compiuto nel volgere di due secoli, tra il XIX e il XX secolo. Nei secoli precedenti al XIX la cura per il mantenimento delle vestigia del passato è consistita nelle riparazioni $\mathrm{e}$ ricomposizioni con finalità utilitaristiche producendo il riuso delle permanenze materiali del passato che erano ricomposte e adattate all'uso vigente. Tale atteggiamento ha caratterizzato l'Antichità e il Medioevo sino agli albori dell'Umanesimo e anche più tardi. Nel Rinascimento la maturazione di un profondo interesse per la conoscenza dell'Antico non ha, nei fatti, generato una diffusa coscienza conservativa, né ha impedito il permanere di un approccio all'uso dei lacerti materiali del passato che non fosse strumentale e selettivo, come avveniva in precedenza: si è ritenuto di dover conservare le sole 


\begin{tabular}{|c|c|}
\hline 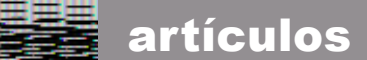 & I progetti della conoscenza e del restauro... \\
\hline
\end{tabular}

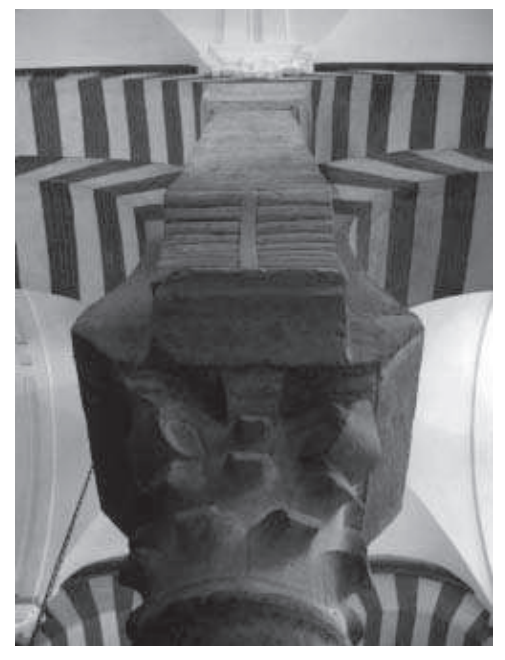

1. La Cattedrale Moschea di Cordoba. Particolare dellordine architettonico dellinterno. Si osserva uno dei pulvini conservati nella forma che evoca una raccolta di rotoli di pergamena.

(Foto di A. Pugliano, maggio 2010. Si ringrazia il Prof. Fernando Moreno Cuadro per aver condotto con perizia e approfondimento la visita illustrando a chi scrive l'importante monumento cordobese).

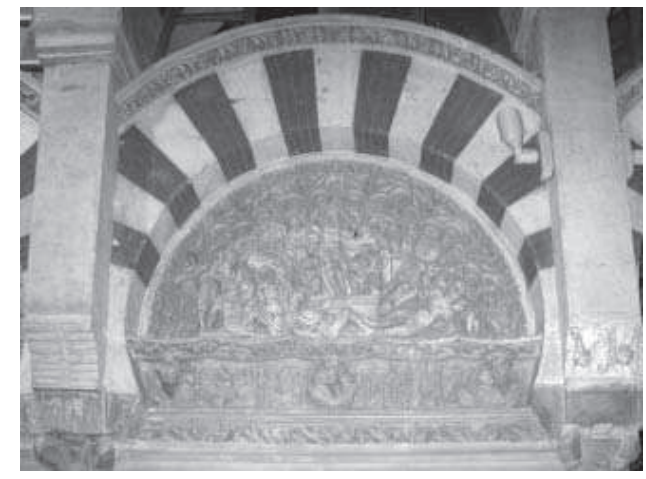

2. La Cattedrale Moschea di Cordoba. Particolare delle addizioni cristiane. Pulvini conservati e pulvini attualizzati in chiave cristiana.

(Foto di A.Pugliano, maggio 2010).

opere congeniali al concetto di Antico che nel frattempo aveva preso forma e di poter procedere alla distruzione d'innumerevoli manufatti non riconosciuti come espressioni di valore culturale, estetico e storico. Gli interventi necessari al mantenimento in uso, all'attualizzazione, dei monumenti da conservare nascevano da un'attenta analisi formale che guidava la ricerca di soluzioni appropriate alla rielaborazione dei caratteri

salienti della preesistenza da assumere all'interno del nuovo programma architettonico. Quest'ultimo, sovente, era basato sulla ricerca di una relazione significante con il passato;un esempio per tutti: la Cattedrale Moschea di Cordoba. Ivi le trasformazioni avvenute consentono, a tutt'oggi,di riconoscere gli ambiti spaziali consegnati al cattolicesimo, percependoli all'interno del tessuto di forme omogeneo nel quale permangono, perfettamente integrate, le evidenze materiali funzionali alla composizione islamica. Espressione di tale condizione è la metodica di adeguamento delle componenti dell'ordine architettonico avvenuto nel tempo con l'edificazione delle addizioni cristiane. Nell'intorno di queste i pulvini che sostengono le superfici intavolate sulle quali imposta l'arco dell'ordine superiore, hanno perso la fisionomia di cartigli - pergamene arrotolate e legate assieme a simulare una raccolta di testi scritti- per acquisire l'immagine di angeli o demoni o per essere ricondotte a semplici mensole di supporto [1-2]. Le superfici intavolate, che sono sostenute dai pulvini a cartiglio, ora sono prive dell'intonaco; tale lacuna compositiva lascia intendere che sia avvenuta l'obliterazione delle iscrizioni 


\begin{tabular}{|c|c|}
\hline 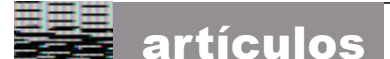 & Antonio Pugliano \\
\hline
\end{tabular}
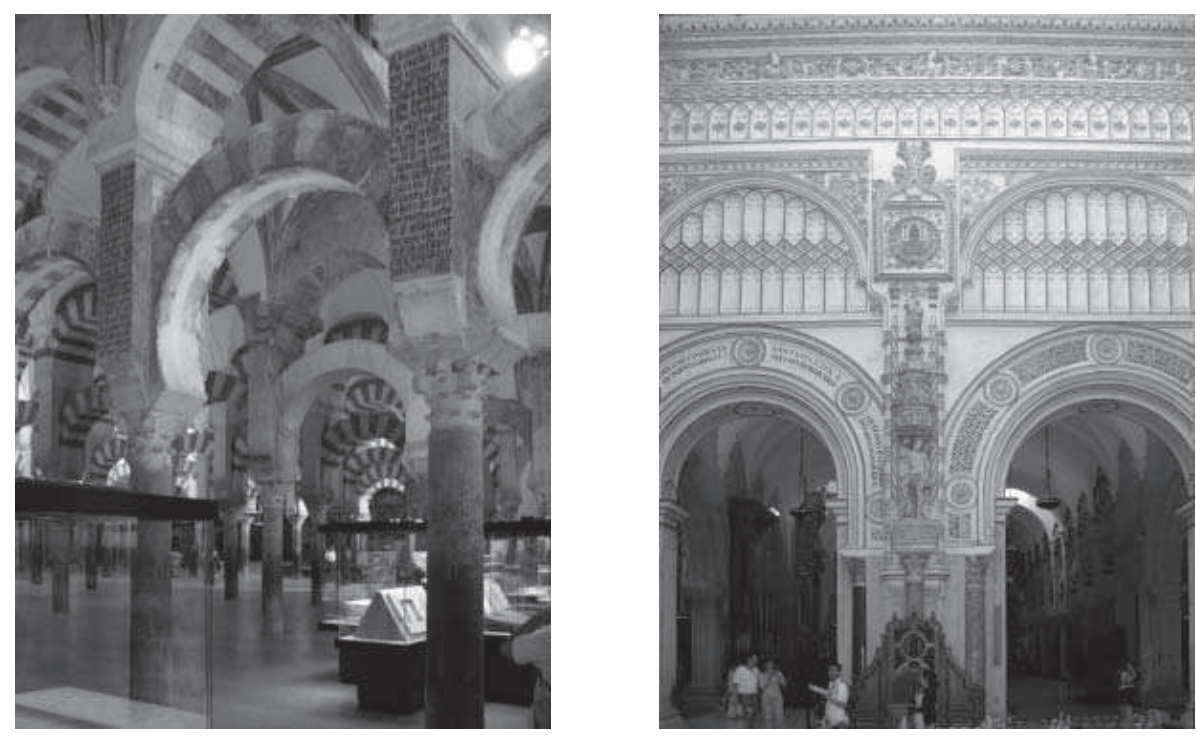

\section{La Cattedrale Moschea di Cordoba. La composizione iniziale dell'ordine architettonico è trasferita al futuro dall'uso delle iscrizioni cristiane. \\ (Foto di A. Pugliano, maggio 2010).}

coraniche recidendo il legame palese tra la forma e il concetto ad essa attinente,

tra il supporto fisico, la pergamena, e l'idea, il verso, da questo trasferita. Tuttavia non è stata sovvertita, intenzionalmente, la composizione complessiva: la trasformazione attuata consente a occhi attenti e a menti informate di cogliere le allusioni ai contenuti ideologici dell'assetto precedente. Negli spazi del connettivo, infatti, è ancora consentito che la narrazione testuale prevalga sulla rappresentazione iconografica e tale assunto è addirittura ribadito, seppure episodicamente, attraverso l'uso d'iscrizioni cristiane [3] ma è negato con forza solo all'interno del recinto di Carlo $V$ dove la consueta composizione architettonica di archi sovrapposti fu reinterpretata formalmente ma soprattutto riformata ideologicamente, opponendo alla chiave aniconica che governa l'intorno,un'emblematica propensione all'espressività iconografica che è ben rappresentata dalla fisicità corporea dei rilievi statuari. [4] 


\begin{tabular}{|c|c|}
\hline 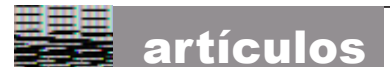 & I progetti della conoscenza e del restauro... \\
\hline
\end{tabular}

\section{il Valore storico, lo Stile e il Restauro.}

L'importanza documentaria dei lacerti del passato divenne preminente nel volgere del XIX secolo, mettendo a frutto le precedenti esperienze degli archeologiarchitetti intenti nello studio tipologico delle architetture antiche e degli storici dell'arte orientati a rintracciare gli elementi caratterizzanti e distintivi delle produzioni artistiche tipiche delle diverse epoche storiche. Si pensi per questo a Giovan Battista Piranesi (Mogliano Veneto 1720, Roma 1778) e alle sue restituzioni dai lacerti delle residenze antiche pompeiane, e le sistemazioni degli storiografi dell'arte, da Giovanni Pietro Bellori (Roma 1613, Roma 1696) a Johan Joachim Winkelmann (Stendal 1717, Trieste 1768), orientate alla definizione di un concetto di stile che esprimesse il genere di appartenenza dei singoli manufatti artistici e, di conseguenza, ne determinasse la potenziale emblematicità. Infine lo stile fu codificato e, secondo l'efficace sintesi prodotta da Etienne Luis Viollet Le Duc (Parigi 1814, Losanna 1879), fu inteso come l'espressione di un ideale fondato su di un principio. Lo stile così definito individuava un sistema coerente di forme storicamente determinate, da analizzare e comprendere nei loro caratteri essenziali affinché potessero essere riproposte e interpretate liberamente nella progettazione di nuove architetture, ispirate a principi antichi. Nella progettazione del restauro, invece,lo stile costituiva un riferimento vincolante le cui espressioni materiche andavano restituite filologicamente attraverso un processo rigoroso di conoscenza e sintesi storica, volto a ripristinare l'unitarietà della consistenza e del significato del manufatto.II limite concettuale di questa posizione teorica fu ben presto individuato: i detrattori considerarono che il fluire del tempo fosse di fatto inarrestabile e che fosse illegittimo, oltre che pretenzioso, pensare di poter riprodurre i processi storici immedesimandosi negli artefici antichi al punto tale da proporre completamenti o trasformazioni genuini.Un siffatto giudizio, seppure di buon senso,tuttavia ha limitato lo sviluppo di un metodo progettuale specifico del restauro che ponesse la conoscenza storica e materiale degli oggettial centro del processo critico di determinazione delle scelte operative.Si trattava di un metodo particolarmente fertile, per il quale la necessità di comprendere approfonditamente le vicende della vita del monumento comportava anche l'analisi del suo contesto culturale di origine, determinando il progredire della ricerca storica. Sul sedime delle esperienze citate si per venne alla maturazione di un concetto di restauro di senso compiuto che possiamo oggi ritenere condivisibile per la convinzione che la valenza documentale delle opere del passato vada salvaguardata in quanto espressione viva della cultura dei popoli e delle nazioni. Fondamentali per questo,furonole elaborazioni teoriche e normative di Ludovico Vitet, (Parigi,1802-1873) di Prospero Merimee, (Parigi 1803, Cannes 1870) e degli Ispettori dei Monumenti Storici Francesi, intenti a produrre, fin dal 1834, l'Inventario delle opere da sottrarre al vandalismo che, non più solo rivoluzionario, era divenuto imprenditoriale e si esercitava sui monumenti medioevali coinvolti pesantemente nei processi di trasformazione urbana tipici della nascente città borghese. Nel volgere del XX secolo il concetto ottocentesco di restauro è stato perfezionato e meditato profondamente 
ponendosi in relazione diretta con la cultura artistica vigente: si è assistito alla crescita del novero delle opere meritevoli di conservazione comprendendo tra queste gli oggetti della tradizione non prodotti da una precisa intenzionalità artistica bensì frutto del sedimentare delle consuetudini locali di cultura materiale. Nel dialogo tra valori storici e valori artistici, quindi, ha trovato spazio il valore antropologico e sociale, espressione anch'essa documentale delle tradizioni locali.

\section{LA PROGETTAZIONE ARCHITETTONICA DEL RESTAURO.}

A causa della sua finalità conservativa, il restauro delle architetture è da ritenersi una disciplina diversa dalla progettazione architettonica della quale, tuttavia, condivide i modi e gli strumenti di espressione: il motivo della diversità è insito nel fatto che il progetto di restauro s'identifica con il progetto della conoscenza. La finalità conservativa, quindi, impone alla progettazione architettonica del restauro l'esercizio di un metodo proprio sostenuto da strumenti adeguati, ma questa sua condizione singolare, al giorno d'oggi, è oggetto di discussione. L'assetto attuale del dibattito interno alla cultura architettonica, infatti, è orientato alla ricerca di una sintesi operativa efficace tra la conservazione delle architetture del passato e la loro traduzione all'oggi in un contesto innovato per forma, linguaggio e funzione; tale ricerca, però, non sembra rivolta alla definizione di una metodologia utile alla prassi progettuale, anzi tende a risolvere nella libera espressività il rapporto con le preesistenze. La pubblicistica di architettura contemporanea documenta una casistica esauriente di esempi reali ove contesti storici ospitano elaborazioni attuali sulle quali è utile soffermarsi a riflettere. Per brevità chi scrive prende in prestito dalla rivista Casabella un'interessante selezione di opere presentate per la loro rappresentatività teorica e metodologica'. L'Autore del saggio sostiene che l'assenza di un comportamento omogeneo frequentato dagli attori della conservazione, siano essi architetti, committenti, o le stesse comunità sociali, dimostri, nei fatti, l'impossibilità attuale di porre in essere una teoria e, quindi, un metodo progettuale efficace e condiviso cui fare riferimento nell'operatività del restauro².

1 DAL CO Francesco, "L'infondatezza del vecchio, l'aleatorietà del nuovo", in: Casabella 754, Electa, Milano Aprile 2007.

La trattazione si apre con la trasformazione del museo Rodin in Parigi (2002-2006 - Pierre-Louis Faloci) ivi è palese indifferenza nei riguardi del linguaggio della preesistenza e lo spazio innovato vince sulla spazialità antica. Segue l'ampliamento del museo Robert Simon in Celle, nella Bassa Sassonia (2005-2006 - Ahrens, Grabenhorst) ove l'obiettivo progettuale esprime la volontà di comporre in chiave dissonante un insieme nel quale la resa estetica complessiva sia dovuta al contrasto tra nuovo e preesistente. In ultimo il completamento del Banco de Espaňa in Madrid (2003-2006 - Rafael Moneo) con il quale si documenta attraverso il completamento dell'edificio storico la tendenza opposta, rivolta alla ricerca di continuità con il carattere della preesistenza attraverso l'assonanza compositiva.

2 "I quattro edifici che presentiamo in questo numero di Casabella, (...) sono stati scelti con cura e con una piccola dose di malizia. Lo abbiamo fatto con l'intento di riproporre all'attenzione dei lettori una questione che non cessa di essere di attualità (...). Si tratta del problema concernente le modalità e i metri di giudizio da adottare per consentire o meno l'inserimento di nuove costruzioni all'interno di complessi storici consolidati, e per favorire o impedire l'utilizzazione per nuovi scopi degli edifici antichi. Come dovrebbe avvenire anche quando si parla di restauro architettonico in senso proprio, affrontando discussioni di questo genere, sarebbe auspicabile i contendenti prendessero concordemente atto del fatto che ogni ragionamento deve scontare 


\begin{tabular}{|c|c|}
\hline 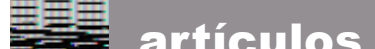 & I progetti della conoscenza e del restauro... \\
\hline
\end{tabular}

Questa affermazione, tuttavia, merita di essere approfondita in chiave storica al fine di rintracciare e valutare, rispettivamente, le origini culturali e la consistenza storica dei diversi comportamenti, per comprendere quanto essi siano appropriati alle istanze della cultura attuale. Due, nella sostanza, sono i modelli comportamentali: un modello prevede l'autonomia, l'altro la congenialità del nuovo rispetto all'antico.Due sono anche i modelli economici cui possono essere ricondotti i diversi atteggiamenti.L'autonomia appare, oggi, frequentata principalmente dalla cultura europea delle regioni più economicamente stabili e consistenti. Esse sono propense a concepire un'idea di progresso basata sulla crescita indefinita, sul primato della produzione massiva di tipo industriale, con carattere globale, della quale sono motore la trasformazione e, soprattutto, l'innovazione intese come le uniche prospettive operative plausibili, peraltro economicamente vantaggiose, da essere elevate al ruolo di valore culturale. La tendenza che chiameremo per brevità continuistica, invece, gode di una consistenza storica e culturale particolarmente ricca nei Paesi mediterranei a tradizione manifatturiera, dove le antiche vocazioni artigianali e artistiche non sono state interrotte dall'industrializzazione ma sono mantenute in vita dalla collettività sviluppando un tessuto di piccola e media imprenditoria impegnata nella ricerca dell'estrema qualità. Questa visione del progresso e del lavoro può essere intesa in chiave etica, come l'espressione tangibile delle proprie radicate e peculiari tradizioni culturalidi valore identitario e, pertanto, da ritenersi un carattere qualificante meritevole di valorizzazione. In tali Paesi è consueta la familiarità con i lacerti del passato, e questo è andato a vantaggio del loro mantenimento nel tempo, seppure attraverso il susseguirsi di processi di attualizzazione.Detta attualizzazionesi è espressa attraverso l'integrazione delle componenti di significato peculiari alle distinte culture che si sono succedute e sovente hanno convissuto nel medesimo contesto materiale. Nella sostanza, il rispetto per il carattere della preesistenza nasce dall'averne conosciuto approfonditamente il valore e il senso; tale conoscenza ha reso agevole il dialogo e la fertile relazione tra culture diverse. La citata visita dell'interno della Cattedrale Moschea di Cordoba chiarisce il concetto. Tale architettura, oggi,è un testo complesso e sfaccettato, ricco di stratificazioni da analizzare e di significati da decifrare per ricomporre la memoria del luogo e trasferirla al futuro nella convinzione che non giovi alla qualità complessiva del monumento alcuna ricomposizione redatta con un linguaggio programmaticamente distante dalle culture che l'hanno prodotto. L'emblematicità della Cattedrale di Cordoba, come di altri monumenti caratterizzati da alto valore simbolico, determina le modalità di restauro da applicare attualmente nella prospettiva della loro valorizzazione. Per tali monumenti il programma conservativo seleziona l'assetto significante e organizza il mantenimento di quest'ultimo imponendo l'assenza di trasformazioni architettoniche che possano contaminarne l'espressione; la ricerca di continuità tra passato e presente si estende anche al futuro: il restauro consiste nella ricomposizione e la conservazione dell'organismo ricomposto è affidata alla manutenzione. La ricomposizione dell'immagine del monumento comporta lo studio approfondito dell'intero organismo architettonico.

l'assenza di valide teorie cui fare riferimento. (...)"

DAL CO Francesco, Op. Cit. pág.3. 
L'efficienza del monumento in termini di espressività deriva da un'attenta, costante e programmata attività di riparazione basata sulla sostituzione delle parti ammalorate con parti riprodotte in analogia formale e materiale. Le parti sostituite debbono essere realizzate in forme non semplificate né parziali, per garantire il trasferimento al futuro delle scelte compositive.Persino le tecniche da utilizzare nel restauro è bene che siano derivate dal magistero antico affinché possano testimoniare la cultura costruttiva e il pensiero tecnologico e scientifico residenti. La progettazione del restauro diviene, quindi, un'attività di ricerca scientifica e storica in grado di definire dati conoscitivi inediti, di estremo valore culturale, che possono divenire l'oggetto della conservazione e, perfino, l'argomento della fruizione del monumento.

\section{LA CONSERVAZIONE E LA VALORIZZAZIONE.}

L'intento di definire concettualmente un modo efficiente ed economicamente sostenibile per coniugare la conservazione alla fruizione dei monumenti e siti archeologici, comporta la necessità di ridiscutere l'approccio teorico e metodologico espresso dalla cultura del restauro negli ultimi decenni, anche recuperando il lascito culturale e metodologico di esperienze precedenti a torto ignorate o malintese dalla disciplina odierna. In Italia, allo stato attuale, è indispensabile chiarire la sostanziale differenza che esiste tra Conservazione e Restauro ribadendo che la prima s'identifica come una finalità, peraltro elementare,e non come una disciplina scientifica o come un metodo operativo. II restauro è lo strumento per il raggiungimento del fine; esso è tanto atto critico quanto atto creativo ${ }^{3}$. La creatività del restauro risiede nel saper individuare e sviluppare le chiavi interpretative più appropriate all'opera, che sono riconosciute come tali sulla base di approfondite analisi critiche. I dati da analizzare riguardano la consistenza storica e materiale dell'opera, il linguaggio formale e tecnico che essa documenta, il legame con il contesto d'origine che essa evoca. I dati vanno riconosciuti nelle tracce materiali ed elaborati progettualmente per determinare le scelte tecniche necessarie. La creatività, quindi, risiede nel saper analizzare il dato dopo averlo rintracciato e selezionato e deve il suo valore all'esercizio di un metodo rigoroso, coerente e assolutamente fertile, tale da determinare il progresso delle conoscenze.Lo strumento con il quale si traduce in atto la conoscenza critica delle opere è il progetto di restauro che è il luogo dell'interpretazione consapevole. Tale approccio genera progetti nei quali l'istruttoria conoscitiva costituisce l'ossatura delle sintesi operative e quest'ultime debbono arrivare, forti della loro consistenza teorica, profondità e coerenza metodologica, a produrre scelte tecniche e di linguaggio singolari, assolutamente

3 Renato Bonelli (Orvieto 1911, 2004), alla metà degli anni 60 del XX secolo, definì il restauro un atto critico e creativo in quanto, alla necessità di riconoscere le qualità artistiche dell'opera, si affianca, di norma, la volontà di intervenire per reintegrare e conservare il suo valore espressivo. Tale valore espressivo risiede nella vera forma ovvero nella forma compiuta del monumento, cioè in quella forma di maggior pregio che va riletta e riproposta, in una parola: liberata. Si osserva che il concetto di <<vera forma >>, da non confondersi con la $<<$ forma originaria $>>$, estende il valore di autenticità dell'opera oltre la materia, sino a comprenderne il contenuto formale. 
pertinenti alle condizioni dell'opera e, pertanto, non definibili a priori secondo alcun orientamento ideologico. La pratica del restaurare, quindi, è un'attività complessa poiché esprime contenuti culturali attraverso scelte tecniche; a questa complessità oggi si risponde con la multidisciplinarietà che, se malintesa, rischia di tradursi in un eccesso di tecnicismo. La prassi descritta appare segnata dal primato della tecnologia sulla scienza, dell'oggettività (spesso più pretesa che reale) sulla capacità critica di elaborazione culturale; in sostanza: il ricorso agli utensili rischia di sostituirsi all'esercizio degli strumenti intellettuali. Tutto ciò lede, negli operatori, la capacità di comprendere e percepire lo stato dei manufatti e, perfino, di preconizzare il danno, com'è necessario nella prassi manutentiva e nella programmazione della conservazione, o di individuare i migliori attrattori da porre in essere nel progetto della valorizzazione.

\section{LA RICHIESTA DI CONSERVAZIONE.}

La conservazione dei beni culturali dipende dalla consapevolezza che si ha del loro valore intrinseco e dall'apprezzamento del bene, che tale consapevolezza induce nella stessa popolazione minuta che abita quel contesto ambientale, oltre che negli amministratori. La conservazione, quindi, si alimenta della cultura diffusa, in altre parole del sistema di convinzioni costruite nel tempo con l'esperienza; esse, una volta indotte o maturate più o meno motivatamente nell'animo dei singoli, divengono espressione del comune sentire dei gruppi da cui è formata una comunità.Vale la pena di riflettere se la multidisciplinarietà e, con essa, l'estremo specialismotecnico, sia la scelta più razionale per creare una crescente richiesta di conoscenza che conduca finalmente all'apprezzamento diffuso delle espressioni d'arte. Per questa finalità, invece, gioca un ruolo fondamentale,l'avvicinamento delle comunità all'arte e la realizzazione della più estesa familiarità con essa. E' fondamentale, quindi, l'istruzione che, nel contesto della formazione degli attori della conservazione, si deve orientare al rafforzamento delle capacità analitiche e operative di figure professionali vocate al coordinamento delle attività conoscitive e tecniche nonché alla diffusione e al radicamento degli esiti culturali della gestione del Patrimonio ${ }^{4}$. La divulgazione delle acquisizioni storiche e critiche, processualie antropologiche, ricavate durante lo studio delle opere da conservare è utile allo scopo citato quanto il loro restauro, beninteso, se esso è orientato alla massima compatibilità filologica.

4 Un'iniziativa scientifica e didattica attenta alla prospettiva indicata sta prendendo forma in Italia anche per opera di chi scrive: attraverso un Programma di Azioni Integrate di Ricerca e Formazione, l'Università Roma Tre e la Soprintendenza Speciale ai Beni Archeologici di Roma-Sede di Ostia intendono progettare la valorizzazione e il restauro del sito archeologico di Ostia per farne un aggiornato Museo della Città Antica utile alla divulgazione dei modi dell'abitare e del costruire nell'antichità. L'aspetto maggiormente interessante e innovativo dell'iniziativa è relativo al fatto che le attività progettuali saranno svolte in forma didattica, all'interno di una Scuola di Specializzazione, ove allievi e docenti, sul campo, collaboreranno nel concepire soluzioni appropriate alle necessità conservative e di valorizzazione dell'importante contesto ambientale. Per maggiori dettagli si guardi a: PUGLIANO, Antonio "Ostia. Un museo della città antica" Ricerche di Storia dell'Arte, $\mathrm{nn}^{\circ}$ 103/104, Roma, 2011, págs. 94-169. 


\section{IL RESTAURO UTILE ALLA VALORIZZAZIONE.}

Attualmente la pratica del restauro è guidata dalla teoria brandiana che ne fornisce una importante definizione nella quale si delineano gli strumenti e il metodo peculiari. Brevemente: il restauro coincide con il momento metodologico per il riconoscimento del valore dell'opera attraverso l'analisi della sua consistenza storica e artistica. La definizione, equilibrata e sintetica, esprime la sintesi perfetta delle innumerevoli esperienze teoriche e pratiche precedenti. Fra queste è senz'altro meritevole di particolare attenzione il contributodi Giuseppe Fiorelli (Napoli,1823;Napoli,1896) ${ }^{5}$ da ritenersi fondamentale per l'approfondimento della metodologia e degli strumenti da applicarsi alla conservazione dei manufatti archeologici. Si tratta di un contributo teorico e metodologico importante che è stato colpevolmente ignorato se non malinteso da storici e restauratori attivi negli ultimi decenni, forse a causa del suo palese radicamento nella prassi operativa ${ }^{6}$. In rapidissima sintesi: nel pensiero di Fiorelli, come del resto avviene nella realtà dei fatti, svolgendo il restauro di un'opera se ne seleziona (consapevolmente ma anche inconsapevolmente) cosa verrà trasferito al futuro nella convinzione che, materialmente, non tutte le informazioni di cui l'opera è portatrice possono essere conservate, pena la perdita materiale del monumento o la riduzione della sua leggibilità e del suo valore di espressione culturale. E' necessario, quindi, che il restauro sia operato nella consapevolezza delle qualità del monumento: la proposta metodologica di Fiorelli si basa sulla considerazione che lo studio preliminare alla scelta degli interventi di restauro deve consistere nell'esame storico-artistico del monumento; la finalità di tale operazione è stabilire quanto debba essere conservato nell'interesse della storia e dell'arte e quanto possa (o debba) essere considerato inessenziale. L'esame storico e artistico deve essere fatto ricorrendo ai documenti storici e approfondendo lo studio diretto del monumento.Si tratta di operare il riconoscimento di valore distinguendo quanto ha vera importanza per la storia o per l'arte e deve essere rispettato, da quanto non ha tanta importanza e può essere variato o soppresso; la fisionomia del monumento delineata per mezzo dell'analisi descritta, e delle scelte a essa consequenziali, è definita da Fiorelli lo stato normale. Individuare lo stato normale, avendo stabilito esattamente ciò che va conservato e, di conseguenza, ciò che va ripensato, significa formulare il giudizio su cosa trasferire al futuro. Operativamente lo stato normale può intendersi, quindi, come una restituzione scientifica, su base storica, della facies più significativa del monumento cui è necessario tendere con il restauro; confrontare tale restituzione con lo stato attuale

5 Numismatico e archeologo ottenne la carica di Ispettore della Soprintendenza e del Museo di Napoli per il Regno delle Due Sicilie. Fu professore di Archeologia presso l'Università di Napoli tra il 1860 e il 1863 e alla costituzione del Regno d'Italia fu Senatore del Regno (1865) nonché Direttore Generale delle Antichità e Belle Arti e diresse gli Scavi archeologici di Pompei, a lui è dovuta la riorganizzazione del sito degli scavi suddivisi in regiones (quartieri) e insulae (isolati) e la numerazione di ciascun ingresso degli edifici. Tra il 1861 e il 1879 curò la redazione del plastico in sughero della città di Pompei conservato a tutt'oggi nel Museo Archeologico Nazionale di Napoli. Nel periodo fra il 1863 e il 1875, condusse la riorganizzazione delle collezioni del Museo Nazionale di Napoli producendo l'inventario generale suddiviso per categorie e classi di materiali, tuttora in uso presso la Soprintendenza Archeologica di Napoli. Fondò a Pompei la Scuola di Archeologia e nel 1875-76 pubblicò la prima guida scientifica della città e diede vita alla rivista di Archeologia Notizie degli Scavi.

6 La figura di Giuseppe Fiorelli è stata rivisitata e valorizzata solo negli ultimi anni e si guardi per questo a: MARCONI Paolo, Il restauro e l'architetto, Marsilio, Venezia 2003. 
consente di individuare, per comparazione, i contesti materiali specifici ove è opportuno intervenire. II metodo e gli strumenti messi a punto da Fiorelli furono diffusi attravero una circolare ministeriale, assumendo così un profilo eminentemente operativo che ha orientato, durante la seconda metà del XIX secolo, l'attività sul campo di archeologici e restauratori, impegnati nella interpretazione accurata di lacerti archeologici da conservare nell'ampiezza dei loro significati storici, artistici e in larga misura anche antropologici .

\section{La Concinnitas e il Riconoscimento di valore.}

L'antecedente del riconoscimento di valore è nella pratica della concinnitas, l'esercizio della quale era dovuto alla competenza degli artefici più colti del passato ${ }^{8}$. Si pensi, per questo, a Francesco Borromini (Francesco Castelli,Bissone, 1599; Roma, 1667) apprezzato oggi per la sua indiscutibile perizia tecnica dalla quale poteva derivare un'estrema libertà compositiva ma che, tra i suoi contemporanei,era ritenuto meritevole di plauso per come sapeva relazionarsi utilmente con le preesistenze. II Borromini concepiva opere segnate da un potente carattere di originalità che derivava dalla rielaborazione inedita di dati acquisiti attraverso gli studi antiquari.In questo senso va riletto l'episodio del restauro antisismico della chiesa abbaziale di San Martino al Cimino, con il dialogo epistolare traVirgilio Spada (Brisighella, 1596; Roma, 1662) architetto della fabbrica e Francesco Borromini, progettista, nell'Ottobre del $1654^{9}$. In tale occasione essi definirono i criteri formali e costruttivi dei nuovi campanili concordando la morfologia esplicitamente ispirata al contesto romanicogotico dell'edificio. La conformità tra parti aggiunte e parti preesistenti fu ricercata nella riproposizione di elementi formali desunti dal repertorio della chiesa e si spinse fino alla qualificazione cromatica delle superfici. In occasione della realizzazione delle cuspidi delle due nuove torri di facciata si registrò un fervore ideativo particolare. Virgilio Spada volle esternare, con maggiore evidenza, la congenialità degli interventi nuovi al carattere della preesistenza, manifestando la volontà di far evolvere la concezione costruttiva seicentesca, tradizionalmente massiva, in un'altra più

7 Governo Italiano, Ministero della Pubblica Istruzione, Direzione Generale delle Antichità e Belle Arti, Circolare 683 bis collegata al D.M. 21 Luglio 1882.

8 Da:DEVOTO,Giacomo e OLI, Gian Carlo: II Devoto-Oli. Vocabolario della lingua italiana, Torino,Le Monnier, 1970 , si estrae il senso attuale del termine concinnitas che, come è noto, proviene da Cicerone: concinnità indica l'armonia risultante dalla corrispondenza delle parti giustapposte; è utile all'interpretazione del ruolo, in architettura, del citato vocabolo la voce Convenance del Dictionnaire Historique d'Architecture di Quatremere de Quincy (Paris 1832). Ivi la convenienzasi considera come attinente alle nozioni di decor individuate da Vitruvio. Osserva Quatremere che, per Vitruvio, il decoro delle architetture, il loro carattere, si esprime nel rapporto, peraltro consequenziale, tra uso degli ordini e natura degli edifici; e, ancora, che esso è assimilabile ai termini accordo e armonia, in relazione all'esistenza di un equilibrato rapporto tra l'interno e l'esterno degli edifici; il termine convenienza identifica così l'abitudine al rispetto per le pratiche consacrate dall'uso nella composizione dell'architettura.

9 CORRADINI,S.Inediti del Borromini nella ristrutturazione di S. Martino, in: Innocenzo X Pamphili: arte e potere nell'età barocca, Roma 1990, e, ancora, FERRI M., PUGLIANO A. La conservazione dei preesistenti caratteri architettonici nei completamenti dell'Abbazia S. Martino al Cimino (secoli XV e XVIII) in: SIMONCINI G. (a cura), La tradizione medioevale nell'architettura italiana -L'ambiente storico -II, Olski, Firenze 1992. 


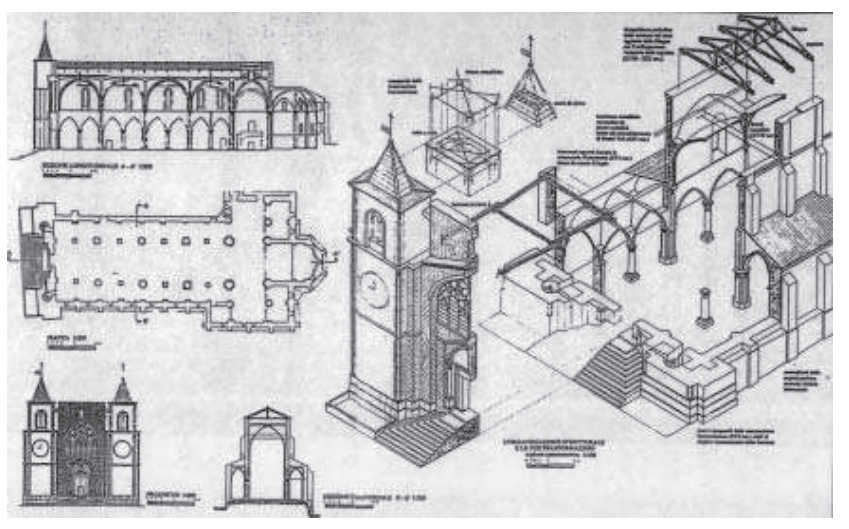

5. Chiesa abbaziale cistercense di San Martino al Cimino (XIII secolo). Gli interventi borrominiani (XVII secolo) hanno completato, secondo concinnitas, l'organismo della chiesa riformando la facciata principale e riparando i danni sismici precedenti (sisma del 1461). La facciata è stata dotata di due torri angolari utili a mitigare il rischio di ribaltamento reso possibile dalle azioni sismiche attese. Le scelte tecniche operate da Borromini appaiono appropriate ed efficienti dimostrando una inattesa consapevolezza del comportamento meccanico degli edifici e dell'abito sismico del luogo.

(Rilievo e restituzione grafica di A. Pugliano).

articolata e gerarchizzata, più affine al magistero gotico. Virgilio Spada concepì tre diverse soluzioni per la realizzazione dei volumi delle piramidi con l'intenzione d'interpretare la morfologia della struttura alla luce dell'assunzione di precisi riferimenti formali e concettuali. Non si trattò della riproposizione in copia di modelli più o meno noti, né di esercitazioni mimetiche nel linguaggio del contesto preesistente bensì si trattò dell'imitazione di caratteri ritenuti peculiari all'architettura medioevale. II primo elemento della nuova composizione concepita da Virgilio Spada fu la colonna gotica da sviluppare su due ordini sovrapposti e comporre con i suoi membretti ai lati; a questo elemento Spada associò strutture diafane e smaterializzate di massima leggerezza percettiva e altrettanta permeabilità alla luce, citando la casistica di edifici, tra cui il campanile di Pisa, che traducevano appropriatamente questa sua interpretazione del gotico. Si possono riconoscere le premesse di un atteggiamento vicino alla teoria dell'arte sviluppata a Roma sul finire del XVII secolo da Giovan Pietro Bellori, sul sedime dei concetti propri alla tradizione neoplatonica. L'operatività artistica, per Bellori, si basava sull'osservazione critica della natura, utile a consentire la conoscenza dei difetti insiti anche in essa, e sull'esperienza del mondo sensibile da cui trarre elementi compositivi finalmente reali e non fantastici. La razionalità, quindi, guida l'artista sia nella fase di analisi, legata all'esercizio della facoltà di scelta, sia in 
fase di sintesi quando si definisce il prodotto artistico sulla base di idee combinatorie ${ }^{10}$. L'interpretazione razionale del monumento imposta dalla ricerca della concinnitas, nel caso di San Martino al Cimino produsse l'individuazione e l'elaborazione originale di alcuni temi riconducibili alla cultura artistica e costruttiva del medioevo. Emerse, parallelamente alla ricerca formale, un notevole approfondimento dell'articolazione strutturale in senso gotico: alla cultura costruttiva medioevale fu riconosciuto il valore di espressione significante prioritaria da conservare e trasferire al futuro anche attraverso la sua riproposizione [5]. La ricerca degli assetti espressivi e qualificanti da conferire alle opere da restaurare è dimostrata da altri esempi, questa volta contemporanei dei quali chi scrive ha conoscenza diretta avendo preso parte ai restauri: il secondo restauro novecentesco della fontana Maggiore di Perugia, di cui si tratta più avanti, e il restauro degli affreschi del San Nicola dei Lorenesi, con la discussione del quale si conclude questa memoria. In entrambi gli oggetti d'arte i restauri hanno evidenziato dati conoscitivi importanti, negati dagli interventi precedenti, ma che sono centrali per la conservazione dei contenuti culturalidei due monumenti.

\section{Il restauro della Fontana Maggiore di Perugia.}

II restauro della Fontana Maggiore di Perugia, con il processo di riconoscimento e di ripristino dello stato normale, si può ritenere un modello di comportamento utile per organizzare la serie di attività progettuali che vanno dall'analisi dei manufatti sino all'esecuzione delle opere, tenendo presenti i requisiti di qualità richiesti oramai alla maggior parte dei processi produttivi ${ }^{11}$.

\section{DEFINIZIONE TIPOLOGICA E TRASFORMAZIONI STORICHE DEL MONUMENTO.}

La Fontana Maggiore di Perugia, come le altre fontane della regione circostante di cui essa è il prototipo (e si guardi agli esempi di Viterbo, Narni, Bevagna, Fabriano) rimanda ai tipi cistercensi riconducibili all'esempio del chiostro del monastero di Poblet in Catalogna; essa, impreziosita da un ricco repertorio di sculture e iscrizioni, è composta da un fusto centrale in bronzo, da cui si attua l'adduzione dell'acqua al sistema tramite zampilli a pressione, e da diversi ordini di vasche in pietra, tra loro sovrapposte, dalle quali l'acqua proveniente dal fusto centrale, tracimando dal bordo del primo recipiente, procede verso il basso condotta da apposite cannelle, sino a raggiungere la vasca sottostante. Realizzata nella seconda metà del XIII secolo (1275-78) come mostra

10 BELLORI, Giovanni Paolo, Vite de' pittori, scultori e architetti moderni, Roma, 1672; e ivi, particolarmente, la premessa sull'ldea della pittura, scultura e architettura.

11 Per gli approfondimenti sui temi della progettazione e dell'esecuzione di tale restauro si guardi a: PUGLIANO, Antonio "Il secondo restauro novecentesco della Fontana Maggiore di Perugia. Note sulla qualità del processo progettuale ed esecutivo nel restauro architettonico", in: Ricerche di Storia dell'Arte $\mathrm{n}^{\circ}$ 65, Roma 1998, págs. 65-102. 
dell'acquedotto del Monte Pacciano, la Fontana di Perugia ha subìto nel corso dei secoli innumerevoli interventi di manutenzione e restauro, alcuni dei quali resi necessari dagli esiti di eventi sismici severi, da sempre consueti in terra umbra. Questi interventi hanno trasferito al nostro secolo un'immagine del monumento fortemente trasformata: il raffinato sistema di assemblaggio delle componenti lapidee concepito dagli scultori Nicola (1215/1220; 1278/1284) e Giovanni (Pisa, 1248 ca; Siena, 1315 ca) Pisano fu oggetto di una prima significativa mutazione nel 1349 quando, per porre rimedio al danno prodotto alla Fontana dal sisma che colpì Perugia in quell'anno, essa venne accomodata sommariamente e, con l'intenzione di ripristinarne la funzionalità, venne dotata di fodere murarie interne ai bacini, necessarie per la tenuta all'acqua. La caratterizzazione cromatica originaria si perse successivamente, con un restauro condotto sotto il pontefice Sisto IV (Francesco Della Rovere, 1471-1484): in quella occasione vennero introdotti molti elementi di sostituzione in travertino, peraltro posti in opera anche in luogo di elementi originariamente realizzati in pietra rossa di Assisi. Nella medesima occasione subì un sensibile impoverimento anche l'esposizione dei significati filosofici, culturali, politici veicolati dalla ricca e articolata compagine scultorea caratteristica del monumento: durante il riassemblaggio delle parti venne confuso l'ordine rigoroso che presiedeva alla successione significativa di statue e di iscrizioni. Altre sostituzioni, ancora in travertino, e altri elementi di confusione nei rimontaggi vennero aggiunti dai restauri del XIX e della metà del XX secolo. II restauro del 1948 fu particolarmente dannoso per il monumento: i restauratori nel riprodurre acriticamente la fisionomia da esso acquisita con le trasformazioni testé elencate, fodere murarie comprese, sottomisero l'architettura della Fontana ad un innaturale comportamento meccanico indotto dalla nuova logica strutturale, eminentemente moderna ed estranea a quella originaria, che essi imposero alla materia che componeva il monumento ${ }^{12}$. Le fodere murarie dei bacini, cui si demandava in parte la funzione strutturale, furono realizzate con malte cementizie, e a esse furono resi solidali i paramenti in pietra; ivi i conci lapidei furono rimontati e murati con malta cementizia interposta tra i giunti d'apparecchio verticali e orizzontali. II tutto ha finito per configurare un organismo appesantito, monolitico ma fragile, privo della duttilità necessaria a superare indenne almeno gli esiti delle fisiologiche sollecitazioni dovute alle escursioni termiche; il trascorrere di cinquanta anni, con le naturali alternanze dei cicli giornalieri e stagionali, ha fatto il resto: già nel 1991 la municipalità perugina si occupò di far redigere un progetto utile a porre rimedio ai segni di rottura che la compagine lapidea presentava in maniera preoccupante. Si trattava, tuttavia, di un progetto concettualmente inadeguato alle potenzialità espressive del monumento: la struttura lapidea sarebbe stata esonerata dalla funzione strutturale ma avrebbe goduto del supporto di una ricchissima struttura reticolare composta da alcune centinaia di aste in titanio cui sarebbero state appese le componenti lapidee. E ancora: la volontà di non mutare l'immagine consueta del monumento, per come era ormai consolidata nella

12 Per la documentazione del restauro del 1948 si guardi a: NICCO FASOLA Giusta, La Fontana di Perugia, Libreria dello Stato, Roma, 1951. 


\begin{tabular}{|c|c|}
\hline$E$ & I progetti della conoscenza e del restauro... \\
\hline
\end{tabular}

memoria collettiva, indusse i progettisti a riprodurre, ancora una volta, il volume delle fodere murarie interne ai bacini ma, intendendo datare all'oggi in maniera evidente tali addizioni, le concepirono come elementi cavi, semplici superfici in vetroresina. Esse, accordate cromaticamente al contesto lapideo sull'estradosso a vista, sarebbero state utili per occultare, al loro interno, il reticolo metallico strutturale. Non è il caso di proseguire oltre con l'illustrazione dei vistosi limiti concettuali e tecnici che trapelano da quanto descritto precedentemente: basta osservare che le peculiarità architettoniche del monumento non sarebbero state comprese e valorizzate adeguatamente e valga l'esempio della totale obliterazione del sistema costruttivo originario che avrebbe lasciato il monumento privo di importanti valenze documentali di carattere storico e tecnico. Tale proposta di progetto, infatti, non trovò nell'opinione pubblica locale alcuna accoglienza benevola, tutt'altro! Secondo un moto dell'animo comune alle più fiere delle popolazioni private improvvisamente del monumento ritenuto emblematico della propria cultura materiale, i perugini richiesero una sostanziale revisione del progetto descritto. Simulare la solidità e, persino, l'integrità del monumento con apparati occulti, con protesi estranee linguisticamente e tecnicamente, non appariva accettabile a quanti, sensatamente, ritenevano che la fontana, come un organismo animato, poteva e doveva essere mantenuta in vita con tutte le sue valenze architettoniche, artistiche, funzionali. Per la revisione del progetto la municipalità di Perugia si avvalse del contributo di una struttura di progettisti composta da architetti restauratori esterni all'Amministrazione comunale; l'attività di tale gruppo di progettisti, negli anni tra il 1996 e il 1999, è descritta di seguito ${ }^{13}$.

\section{IL CANTIERE DI ANALISI E D'INTERVENTO.}

La metodologia posta in essere dai nuovi progettisti ha delineato una successione ordinata di azioni necessarie alla comprensione estesa ed esauriente delle peculiarità del manufatto: la ricognizione sulle vicende storiche del monumento è stata associata alla conoscenza delle sue caratteristiche materiali e dello stato di conservazione. II progetto ha inteso conservare i significati formali, tipologici e costruttivi del monumento attraverso il riconoscimento e la valorizzazione della concezione architettonica originaria.

\section{IL Riconoscimento dello stato NORMALE DEL MONUMENTO.}

L'impegno iniziale dei progettisti riguardò la conoscenza della legge compositiva che regolava la compagine materiale della fontana, iniziando ad analizzare i sistemi

13 L'incarico di consulenza progettuale è stato affidato con delibera della Giunta Municipale di Perugia, n. 1712 del 13.06.1996, a Paolo Marconi ed esteso, con delibera n. 1063 del 19.11.1998, ad Antonio Pugliano. Hanno partecipato alla redazione del progetto, collaborando con i progettisti incaricati, Carlo Baggio per le verifiche di calcolo, Paola De Rosa e Fabrizio Pompozzi per la grafica informatizzata. 


\begin{tabular}{|c|c|}
\hline 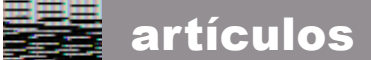 & Antonio Pugliano \\
\hline
\end{tabular}

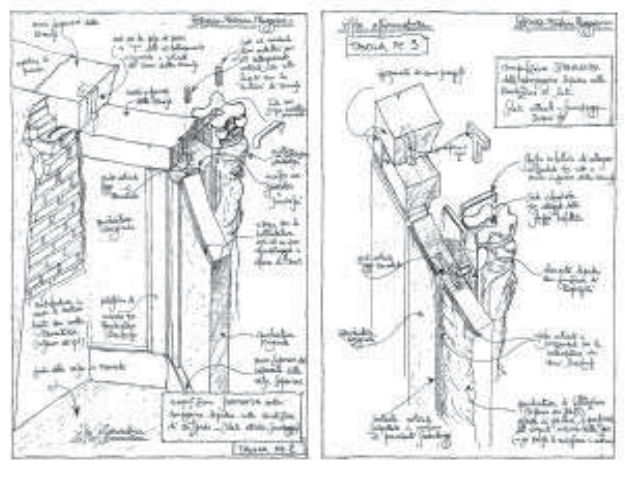

6. La Fontana Maggiore di Perugia (XIII secolo). Documentazione dell'assetto tecnologico tipico del monumento e delle estese trasformazioni indotte dal pernicioso restauro del 1948. Si osserva la muratura in mattoni $e$ malta cementizia realizzata allinterno della vasca superiore e l'abolizione degli antichi sistemi metallici $d i$ collegamento tra le componenti lapidee.

(PUGLIANO A. Taccuino di cantiere 1999).

\section{La Fontana Maggiore di Perugia (XIII secolo). Restituzione congetturale, dai lacerti, della composizione strutturale antica da ripristinare con il restauro attuale. A destra, descrizione sintetica del comportamento meccanico acquisito dalla Fontana con i restauri del 1948; $i$ cinematismi di rottura verranno aboliti dalla ricomposizione della struttura antica. \\ (PUGLIANO A. Taccuino di cantiere 1999).}

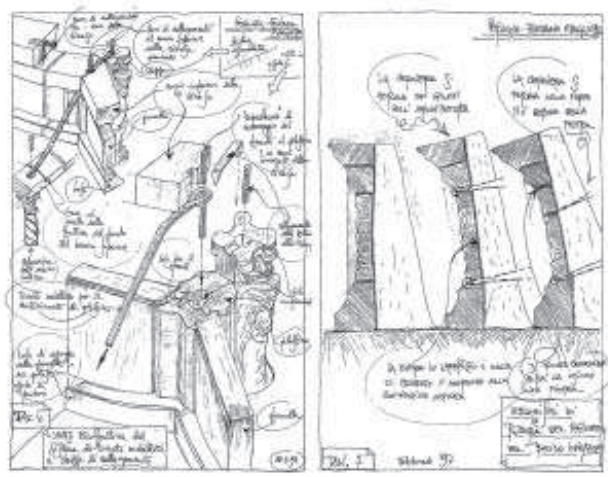

di collegamento tra gli elementi lapidei e la loro gerarchia funzionale [6]. Quest'ultima prevedeva originariamente l'unione di parti, autonome e di geometria determinata, all'interno di una composizione gerarchicamente preordinata; l'organismo era stato assemblato facendo ricorso a raffinate inserzioni di un elemento nell'altro. L'originario sistema di unioni tra le parti era garante di una certa solidità dell'organismo e, insieme, della sua necessaria duttilità, ma le caratteristiche geometriche dei conci utili a tale funzione erano in parte andate perdute e ciò aveva comportato la diminuzione dell'effettiva loro capacità di essere solidali e connessi. Fu evidente ai progettisti che la reintegrazione delle componenti lapidee strutturalmente inefficienti, avrebbe posto in essere i presupposti materiali per l'efficacia del comportamento strutturale dell'organismo restaurato. Al fine di ripristinare un accettabile comportamento meccanico, e per rendere agevole in futuro la pratica manutentiva, si è ritenuto di dover procedere allo smontaggio pressoché integrale dell'organismo lapideo ricorrendo alla rimozione delle addizioni murarie posticce e, soprattutto, all'eliminazione del cemento colato nei giunti dell'apparecchio lapideo per ottenere l'effettiva liberazione delle sue componenti dal pernicioso legame che ne determinava la fragilità [7]. La procedura di smontaggio 
8. La Fontana Maggiore di Perugia (XIII secolo). Il progetto esecutivo. A destra il riconoscimento dell'antico processo di assemblaggio delle componenti lapidee da riprodurre per lo smontaggio accurato del monumento. A sinistra lo Stato Normale del monumento da ripristinare attraverso il restauro.

(PUGLIANO A. Taccuino di cantiere 1999).
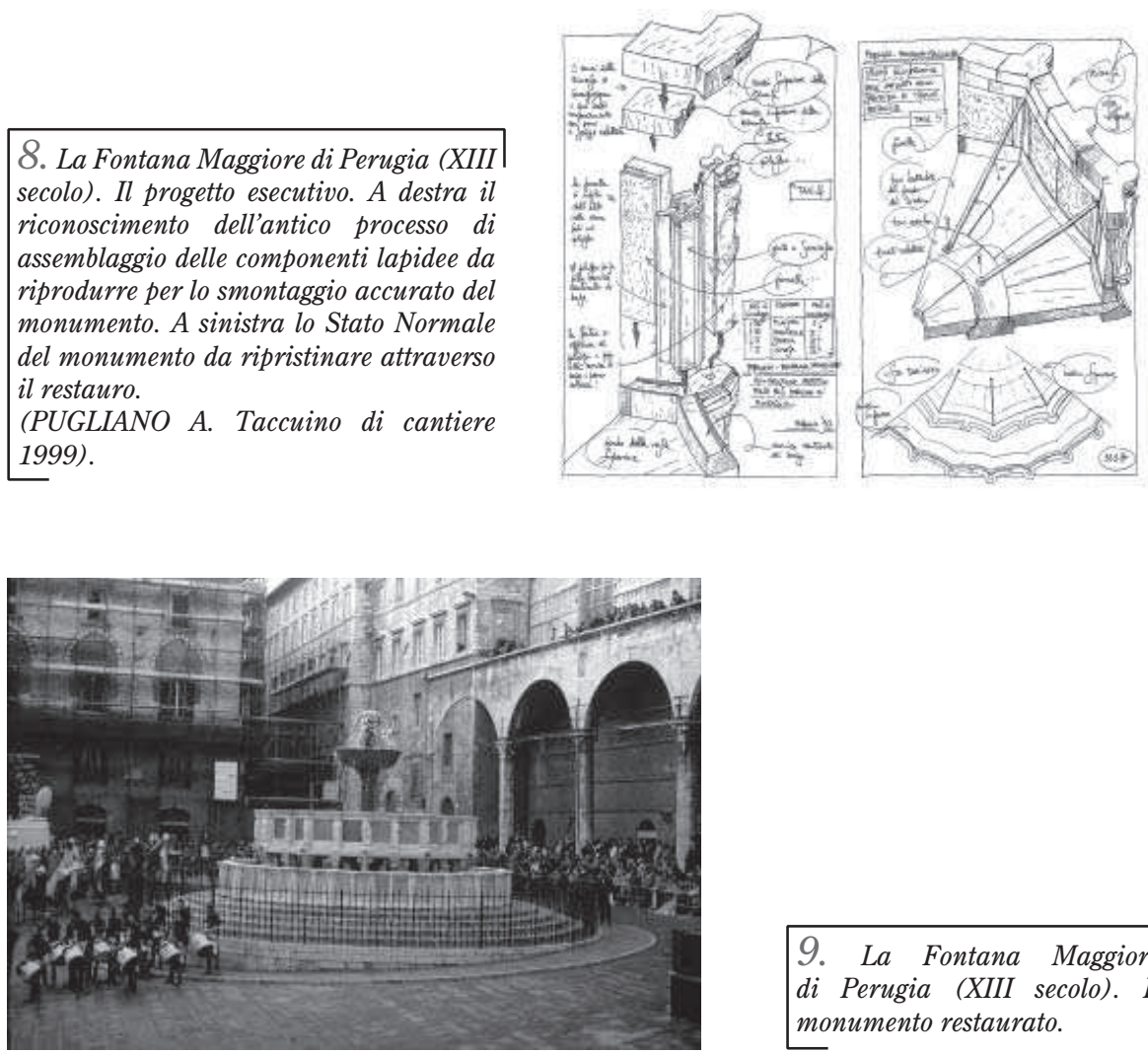

9. La Fontana Maggiore di Perugia (XIII secolo). Il monumento restaurato.

attuata fusvolta in tutta sicurezza essendo congeniale alla struttura in quantosi ispirava, invertendone la successione, al procedimento originario di montaggio [8].

\section{IL RIPRISTINO DELLA COMPOSIZIONE ORIGINARIA.}

Per l'efficace comportamento meccanico dell'organismo riassemblato si è proceduto alla tassellatura delle componenti lapidee danneggiate eal ripristino dei dodici tiranti metallici inclinati che furono riconosciuti come appartenenti alla composizione strutturale concepita dagli antichi artefici. La fisionomia strutturale sino ad ora descritta reinterpretava, in termini di forte affinità, la concezione architettonica di Nicola e Giovanni Pisano che tornava, finalmente, a essere leggibile. Nel giugno 1999, la Fontana è stata restituita ai perugini con una fisionomia architettonica appropriata oltre che nella sua piena funzionalità strutturale e idraulica [9]. 
Il Restauro dell'affresco absidale della chiesa romana di San Nicola dei LORENESI.

II monumento di cui si tratta è un edificio di antica origine che ha subito nel tempo molte importanti trasformazioni tipiche degli insediamenti cristiani di età tardo antica in Roma. Originariamente occupò una campata del Circus Agonalis (il sostrato dell'odierna Piazza Navona) al momento in cui esso cadde in disuso dal IV secolo d.C; fu estesamente ricomposta e ampliata tra il XVI secolo e il XVII per essere rinnovata nella decorazione interna tra i secoli XVIII e il XIX ${ }^{14}$.

\section{VALENZE STORICHE E CULTURALI, LORO ESPRESSIONE NEL MONUMENTO.}

La vita della Chiesa è legata alle sorti della comunità francese nella città di Roma: dal XVI secolo, la chiesa di Saint-Nicolas in Agoneè recensita tra le cinque pertinenti a detta collettività e le vicende dei secoli successivi testimoniano il processo di riconoscimento della singolarità nazionale della comunità lorenese in seno alla più estesa comunità d'oltralpe. Anche nella scelta della Chiesa di San Nicola in Agone si espresse un significato politico: la localizzazione fu strategica e, condividendo il sito della chiesa di Santa Maria dell'Anima appartenente alla comunità tedesca di Roma, si intese rammentare la storia della Lorena, l'antica Lotharingia,la regione del confine anche culturale, del bilinguismo franco tedesco. Nel 1622 la comunità dei Lorenesi a Roma, già fortemente strutturata, chiese e ottenne dal Papa Gregorio XV (Alessandro Ludovisi, 1621-1623) il possesso della chiesa parrocchiale di San Nicola in Agone per poter evolvere dalla semplice rappresentanza di una cappella in San Luigi dei Francesi che era la loro dotazione da circa un secolo. La Chiesa di San Nicola, dal 1633 al 1636, fu estesamente trasformata; per opera di un architetto di nome Francesco Giardino ${ }^{15}$ fu dotata di una volta nella navata e sormontata da una cupola con lanternino. Plausibilmente, la nuova facciata in pietra fu realizzata riutilizzando il travertino proveniente dai lacerti dell'antico circo agonale all'interno del quale si collocava la cripta della chiesa [10]. II secolo successivofu caratterizzato dall'impegno per la qualificazione dell'interno dell'edificio. Attraverso l'imposizione di suntuose rappresentazioni a tema, in affresco

14 Per le vicende storiche della chiesa di San Nicola dei Lorenesi si guardi a:

LA CROIX Pierre, "Mémoire historique sur les institutions de la France à Rome puisé dans les arcbives et autres documents lo plupart Inédits" - 2ème édition; éd. Imprimerle Editrice Romana, Rome (1892).

BRUGNOLA Gildo "Les Pieux. Etablissements de la France à Rome et à Lorette" - éd. Typis Polyglottis Vaticanis, Vatican (1956).

De DUMAST Maxime “L'église Saint-Nicolas-des-Lorrains à Rome”- éd. In.Gra.Ro, Rome (s.d.)

VIOLETTE Patrick "La décoration de l'église de Saint-Nicolas-des-Lorrains (1623-1870)"; in Les fondations nationales dans la Rome pontificale éd. Ecole française de Rome,Rome (I 981).

COLLIN Hubert: "La Rome des papes et l'église Saint-Nicolas-des-Lonains", in: Plaidoyer pour l'église SaintNicolas-des-Lorrains à Rome - éd. Association des Amis de Saint-Nicolas-des-Lorrains, Nancy (1989).

ROCHETTE Jean Claude, Rome-Eglise Saint-Nicolas-Des-Lorrains, Relazione Dattiloscritta cons. In: Archives de Saint-Nicolas-Des Lorrains, 1991, Roma.

15 ROCHETTE Jean Claude, Op. Cit. 4- Analyse Historique, Archeologique et Chronologie (Architecture et decors interieurs) pág. 10. 


\begin{tabular}{|c|c|}
\hline EE & I progetti della conoscenza e del restauro... \\
\hline
\end{tabular}

10. La chiesa di San Nicola dei Lorenesi in Roma (XVIII secolo). Rilievo architettonico della chiesa con $i$ suoi annessi e documentazione del posizionamento dell'edificio sul sedime dell'antico Circus Agonalis; documentazione della volumetria attuale del monumento.

(Rilievi ed elaborazioni grafiche di Valeria Bertuccio, Giulia Di Francesco, Simone Diaz per PUGLIANO A., Chiesa di San Nicola dei Lorenesi. Contributi analitici $e$ operativi al Restauro degli affreschi della parete absidale (sec. XVIII). Consulenza scientifica progettuale, Roma 2006).
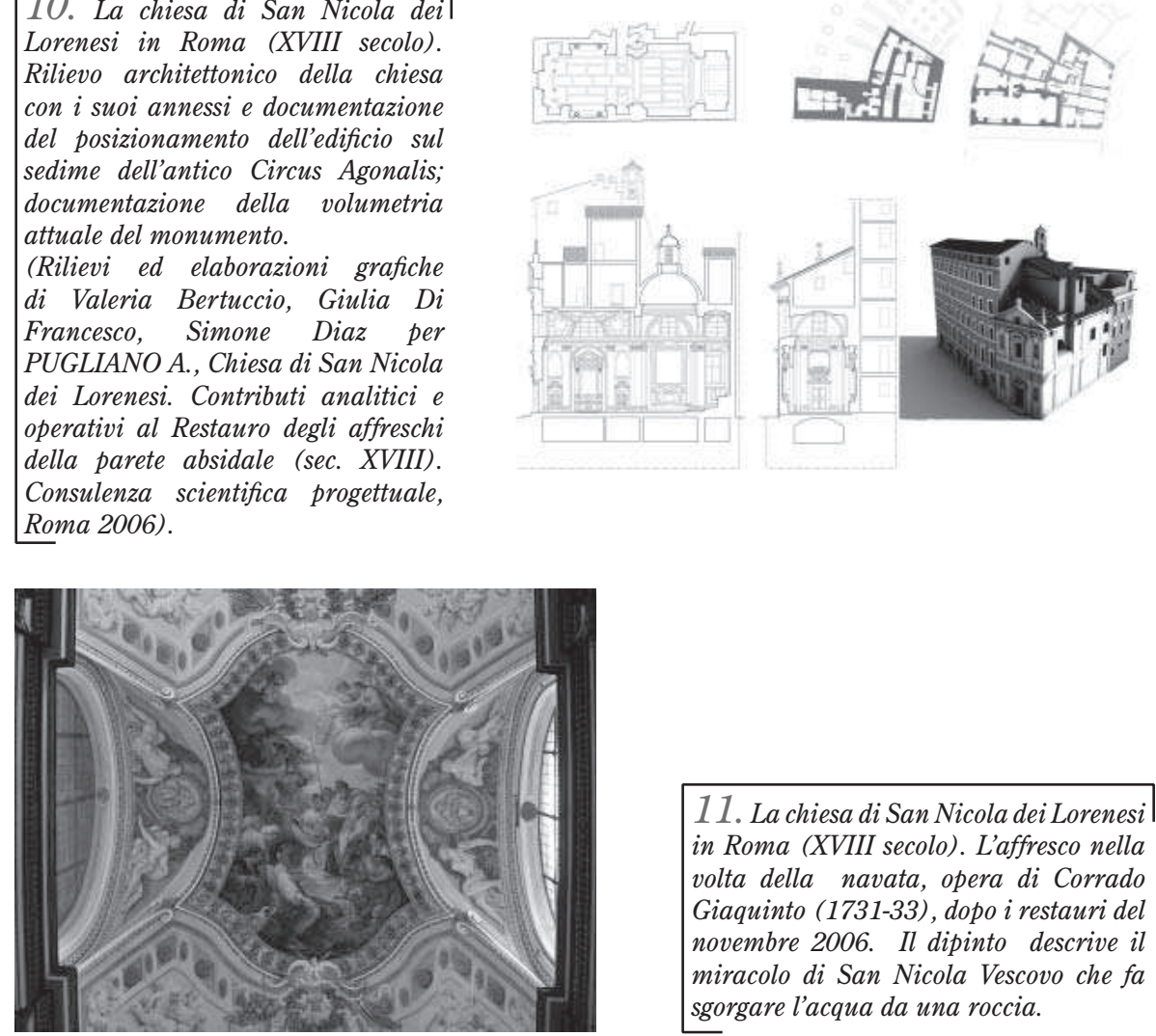

11. La chiesa di San Nicola dei Lorenesi
in Roma (XVIII secolo). L'affresco nella
volta della navata, opera di Corrado
Giaquinto (1731-33), dopo i restauri del
novembre 2006. Il dipinto descrive il
miracolo di San Nicola Vescovo che fa
sgorgare l'acqua da una roccia.

"à l'italienne", si trasformò la precedente composizione ispirata alla figuratività barocca di pure superfici imbiancate. Gli affreschi settecenteschi alle volte e alla cupola sono per lo più opere pregevoli; alcune, datate al 1731, sono tra le prime a essere prodotte da un noto artista italiano di levatura internazionale del XVIII secolo, Corrado Giacquinto (Molfetta 1703, Napoli 1763) di formazione napoletana, che fu attivo a Roma, a Torino, nonché a Mafra in Portogallo, e fu molto presente a Madrid e a Segovia per volere di Carlo III e a Napoli per conto di Ferdinando IV di Borbone [11]. L'ultimo affresco a essere realizzato per il Giubileo del 1750, fu il trompe l'oeil che incornicia l'altare maggiore, dipinto attribuito a Pietro Rossini e Giuseppe Silvestri ${ }^{16}$. Con la soppressione dei Lieux pies de Rome a opera della Repubblica Francese, nel 1799 la chiesa venne privata della sua funzione che riacquistò solo tra il 1804 e il 1816 con la reintroduzione del culto e la rifondazione degli statuti delle comunità francesi di Roma. II XIX fu un secolo di restauri e, tra questi, si annoverano gli estesi interventi di ritocco all'affresco

16 ROCHETTE Jean Claude, Op.Cit. 4 - pág. 11. 
attorno all'altare maggiore condotti nel $1825^{17}$ per il giubileo sotto Leone XII (Annibale Sermattei della Genga, 1823-1829) sui quali si sono concentrate le ricerche svolte da chi scrive in occasione degli ultimi restauri.

\section{RESTAURI RECENTI E L'INDIVIDUAZIONE DEI DATI STORICI DA ESPORRE.}

Sono datati al 2006 i restauri delle decorazioni pittoriche interne al San Nicola dei Lorenesi ${ }^{18}$. I restauri accurati hanno restituito forza e leggibilità alla ricca compagine d'intonaci marmolidei, di stucchi indorati e di affreschi trompe l'oeil, ma, essendo orientati soprattutto alla conservazione oggettiva e materiale dell'assetto vigente, rischiavano di lasciare incompreso un tema rilevantedi carattere storiografico, dimostratoda alcune trasformazioni introdotte nel tempo a danno della qualità della decorazione pittorica. Particolarmente imbarazzante per i Conservatori della chiesa è stataritenuta l'inefficacia geometrica e formale che caratterizzava la composizione dell'affresco a cornice dell'altare maggiore rappresentante, a trompe l'oeil, una mossa e articolata edicola architettonica [12]. Ivi le componenti dell'ordine architettonico apparivano rilavorate in maniera grossolana e frettolosa con palesi debolezze stilistiche, di linguaggio e composizione che avevano estesamente degradato l'assetto compositivo riformando la visione prospettica e correggendo di conseguenza la fisionomia architettonica degli ordini dipinti. II tutto sembrava generato dalla necessità di riformare la parte sommitale dell'affresco e, soprattutto, il sistema costituito dai dipinti della Croce e dalle volte a essa sovrapposte [13].

\section{I preliminari analiticl. Critica dello stato attuale e deI lacerti rinvenuti.}

Attraverso l'esame dell'assetto attuale dei dipinti sono emersidue temi di riflessione: l'incongruenza compositiva tra la croce, la direzione dei raggi della gloria e l'architettura del contesto voltato, plausibilmente anch'esso rimaneggiato; la scarsa qualità prospettica e formale dell'immagine della croce, ove si osservava che non apparivano sufficientemente caratterizzate prospetticamente sia la traversa e le sue testate terminali, sia il corpo con le sue estremità. Il dettaglio delle componenti, inoltre, era apparso generalmente confuso e lacunoso.L'appoggio della croce attuale appariva rilavorato e aveva assunto una forma assolutamente sommaria. In origine si poneva un vaso contornato da godroni, in analogia formale con il fonte battesimale rappresentato nella composizione scultorea a stucco sita sulla parete di sinistra della chiesa. Detto vaso era sormontato da foglie con frappature di cui restava l'esteso lacerto documentato dalle incisioni sull'intonaco.

17 Ibídem - pág. 12.

18 PUGLIANO Antonio, Chiesa di San Nicola dei Lorenesi. Contributi analitici e operativi al Restauro degli affreschi della parete absidale (sec. XVIII). Consulenza scientifica progettualealla restauratrice Gabriella De Monte (Impresa S.E.I. 1983 S.n.c. Roma;committenti Ministére de la Culture e de la Comunication, Ambassade de la France prés la Saint-Siége, Ambassade de la France en Italie, Pieux Etablissements de la France à Roma et à Lorette.). Roma, novembre 2006. 


\begin{tabular}{|c|c|}
\hline 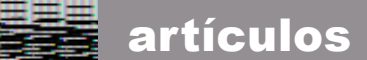 & I progetti della conoscenza e del restauro... \\
\hline
\end{tabular}

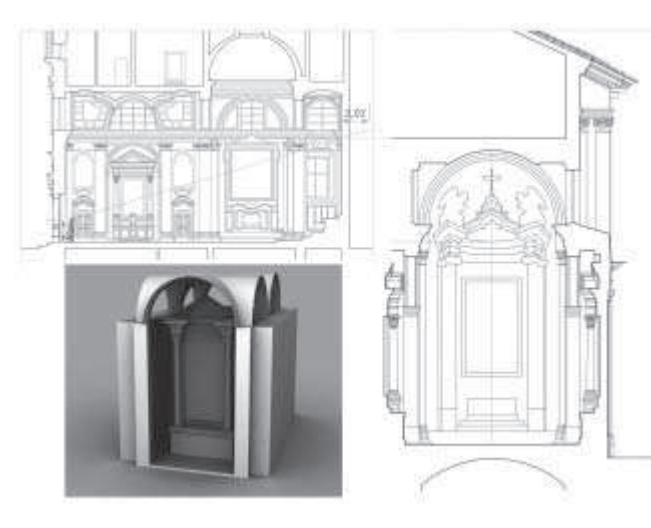

12. La chiesa di San Nicola dei Lorenesi in Roma (XVIII secolo). Interpretazione geometrica dell'affresco di Pietro Rossini e Giuseppe Silvestri (1750) posto nell'abside a coronamento dell'altare maggiore. Il dipinto prospettico rappresenta l'altare come contenuto in un'architettura absidale composta di due campate coperte da volte a crociera.

(Rilievi ed elaborazioni grafiche di Valeria Bertuccio, Giulia Di Francesco, Simone Diaz per PUGLIANO A. Cit., Roma 2006).

\section{RICONOSCIMENTO DAI LACERTI DELLA} FISIONOMIA DELLA CROCE DI RIPRISTINO.

L'individuazione e l'esame dei lacerti rintracciabili nel contesto del disegno da restaurare, sui quali basare le ipotesi di ricomposizione, è avvenuta attraverso riprese fotografiche di appropriata risoluzione che hanno reso palesi alcune tracce importanti. In particolare il lacerto di una traversa, più grande dell'attuale, appariva fortemente dettagliato; la medesima foto consentiva di isolare altre linee interessanti per la composizione generale.

\section{LE SINTESI OPERATIVE. ELABORAZIONE DEL PROGETTO DI RESTAURO.}

L'elaborazione del progetto di restauroè iniziata con la documentazione delle dimensioni e delle forme della croce esistente dalla quale derivare la fisionomia dalla croce da ripristinare. Sono state delineate due fisionomie possibili: per la definizione della prima ipotesi di croce di ripristino, si è rielaborata la fisionomia esistente rendendola coerente con alcuni dei segni rintracciati; la traversa e il corpo della croce 

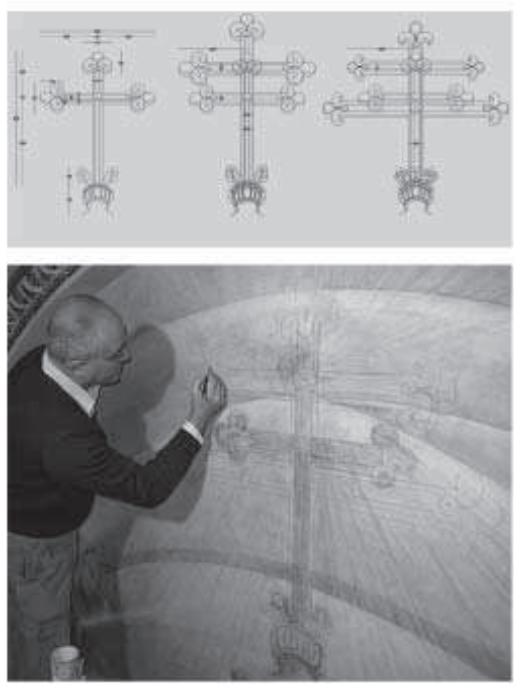

14. La chiesa di San Nicola dei Lorenesi in Roma (XVIII secolo). Il restauro dell'affresco. Le sintesi di progetto finalizzate all'individuazione dello stato normale della Croce (sopra): il rilievo della croce $d a$ restaurare guida la definizione delle due fisionomie architettoniche da verificare in relazione alla congruenza di ciascuna con l'intorno che la deve ospitare. L'esecuzione del progetto (sotto) è consistita nella riproduzione della croce di ripristino direttamente sullintonaco dando corpo alle tracce e reintegrando le lacune.

(PUGLIANO A. Cit., Roma 2006). sono stati determinati sulla base delle dimensioni documentate dai lacerti. La croce così definita, pur coerente con la direzione dei raggi della gloria, non godeva di una appropriata giacitura nel contesto dell'architettura dipinta. La seconda ipotesi ha sviluppato una fisionomia inedita (a due traverse) coerente con la totalità dei segni rintracciati. La croce è coerente con la direzione dei raggi della gloria, gode di una appropriata giacitura e di un efficace assetto prospettico. Le qualità di questa fisionomia ha determinato la scelta della sua riproduzione [14].

L'ESECUZIONE DEL PROGETTO DI RESTAURO.

Si è proceduto alla ripresa diretta dei segni presenti sull'intonaco procedendo, poi, alla reintegrazione formale, con i dovuti aggiustamenti di carattere prospettico, delle lacune presenti. II ritocco delle incisioni nell'intonaco e l'osservazione del repertorio di elementi analoghi presenti nella chiesa ha suggerito la fisionomia complessiva della reintegrazione. L'interpretazione formale del vaso con godroni e foglie alla base della croce è stata in qualche misura più libera: essendo molto lacunoso e confuso il disegno esistente essa ha finito per acquisire il carattere degli elementi pittorici dell'intorno più prossimo [15]. II metodo sperimentato in occasione del restauro della croce è stato applicato alla definizione della fisionomia da assegnare alla compagine fortemente manomessa dell'ordine architettonico. II restauro ultimato ha riposto in essere la Croce a doppia traversa, utile a documentare, con coerenza, la genesi e la linea di sviluppo culturale del monumento [16]. II giudizio operato dai restauratori contemporanei ha, quindi, selezionato i contenuti da conservare attraverso il monumento, consegnando alla sola documentazione storica di carattere letterario la memoria del maldestro restauro del 1825. 


\begin{tabular}{|c|c|}
\hline 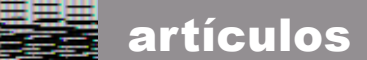 & I progetti della conoscenza e del restauro... \\
\hline
\end{tabular}

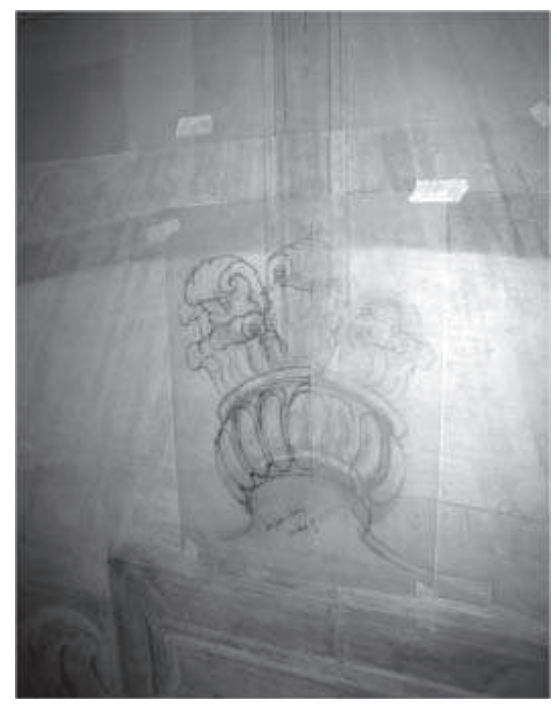

15. La chiesa di San Nicola dei Lorenesi in Roma (XVIII secolo). Il restauro dell'affresco. La definizione dell'assetto formale di restauro delineato per la base di appoggio della croce di ripristino.

(PUGLIANO A. Cit., Roma 2006).

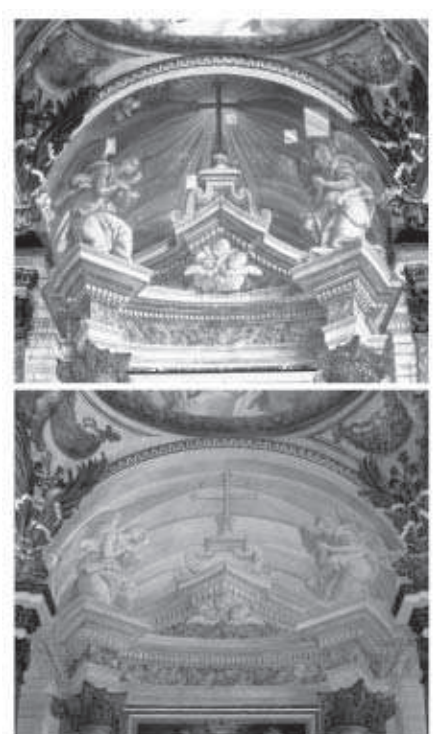

16. La chiesa di San Nicola dei Lorenesi in Roma (XVIII secolo). Le fisionomie architettoniche della parte sommitale dell'affresco absidale, prima (sopra) e dopo (sotto) il restauro del 2006.

(PUGLIANO A. Cit., Roma 2006).

\section{Per concludere.}

La casistica degli esempi ha presentato interventi di restauro orientati a reintegrare l'immagine di monumenti malintesi ricomponendone la storia in una visione critica e non pretestuosamente oggettiva. In tali restauri di valorizzazione i significati riscoperti compongono una narrazione storica fruibile che rafforza l'interesse delle comunità per la conoscenza della culturale del proprio passato. Tale rinnovato interesse può essere alla base di un modello di sviluppo economico più adatto e sostenibile, di quanto sia l'industrializzazione, a contesti territoriali caratterizzati da importanti ed estese permanenze materiali di valore storico. La valorizzazione di beni culturali pertanto, comporta un cambiamento di prospettiva nella prassi della conservazione: il restauro necessita di evolvere e tornare ad esercitare le sue peculiarità metodologiche e critiche per risultare, di nuovo, una pratica progettuale distinguibile dalla progettazione architettonica della quale, nei fatti, ha finito per condividere la vocazione alla libertà espressiva piuttosto che alla ricerca e alla sintesi conoscitiva di carattere storico e culturale. 
